\title{
Prognostic models for predicting recurrence and survival in women with endometrial cancer
}

DOI:

10.1002/14651858.cd014625

\section{Document Version}

Final published version

Link to publication record in Manchester Research Explorer

\section{Citation for published version (APA):}

Wan, Y-L., El Sayed , I., Walker, T., Russell, B., Badrick, E., McAleenan, A., Edmondson, R., \& Crosbie, E. (2021). Prognostic models for predicting recurrence and survival in women with endometrial cancer. The Cochrane Library, 2021(6), [CD014625]. https://doi.org/10.1002/14651858.cd014625

\section{Published in:}

The Cochrane Library

\section{Citing this paper}

Please note that where the full-text provided on Manchester Research Explorer is the Author Accepted Manuscript or Proof version this may differ from the final Published version. If citing, it is advised that you check and use the publisher's definitive version.

\section{General rights}

Copyright and moral rights for the publications made accessible in the Research Explorer are retained by the authors and/or other copyright owners and it is a condition of accessing publications that users recognise and abide by the legal requirements associated with these rights.

\section{Takedown policy}

If you believe that this document breaches copyright please refer to the University of Manchester's Takedown Procedures [http://man.ac.uk/04Y6Bo] or contact uml.scholarlycommunications@manchester.ac.uk providing relevant details, so we can investigate your claim.

\section{OPEN ACCESS}


Cochrane Database of Systematic Reviews

\section{Prognostic models for predicting recurrence and survival in women with endometrial cancer (Protocol)}

Wan YL, El Sayed I, Walker TDJ, Russell B, Badrick E, McAleenan A, Edmondson R, Crosbie EJ

Wan YL, El Sayed I, Walker TDJ, Russell B, Badrick E, McAleenan A, Edmondson R, Crosbie EJ.

Prognostic models for predicting recurrence and survival in women with endometrial cancer (Protocol).

Cochrane Database of Systematic Reviews 2021, Issue 6. Art. No.: CD014625.

DOI: 10.1002/14651858.CD014625.

www.cochranelibrary.com

Prognostic models for predicting recurrence and survival in women with endometrial cancer (Protocol) 
TABLE OF CONTENTS

HEADER 1

ABSTRACT

BACKGROUND

OBJECTIVES

METHODS

ACKNOWLEDGEMENTS

REFERENCES

ADDITIONAL TABLES

APPENDICES

CONTRIBUTIONS OF AUTHORS

DECLARATIONS OF INTEREST

SOURCES OF SUPPORT 
[Prognosis Protocol]

\section{Prognostic models for predicting recurrence and survival in women with endometrial cancer}

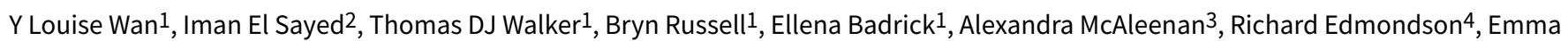
J Crosbie 1

1Division of Cancer Sciences, Faculty of Biology, Medicine and Health, The University of Manchester, Manchester, UK. ${ }^{2}$ Department of Biomedical Informatics and Medical Statistics, Medical Research Institute, Alexandria University, Alexandria, Egypt. ${ }^{3}$ Population Health Sciences, Bristol Medical School, University of Bristol, Bristol, UK. ${ }^{4}$ Division of Cancer Sciences, Faculty of Biology, Medicine and Health, The University of Manchester, Manchester, UK

Contact address: Y Louise Wan, ywan@doctors.org.uk, yee-loi.wan@manchester.ac.uk.

Editorial group: Cochrane Gynaecological, Neuro-oncology and Orphan Cancer Group.

Publication status and date: New, published in Issue 6, 2021.

Citation: Wan YL, El Sayed I, Walker TDJ, Russell B, Badrick E, McAleenan A, Edmondson R, Crosbie EJ. Prognostic models for predicting recurrence and survival in women with endometrial cancer (Protocol). Cochrane Database of Systematic Reviews 2021, Issue 6. Art. No.: CD014625. DOI: 10.1002/14651858.CD014625.

Copyright $\odot 2021$ The Cochrane Collaboration. Published by John Wiley \& Sons, Ltd.

\section{A B S T R A C T}

\section{Objectives}

This is a protocol for a Cochrane Review (prognosis). The objectives are as follows:

To review all prognostic models that combine two or more clinical, histological or molecular variables, or a combination of these variables, to provide an individualised assessment of risk of recurrence or death from disease and evaluate their performance to predict these outcomes in people undergoing curative treatment for endometrial cancer. 


\section{B A C K G R O U N D}

\section{Description of the health condition and context}

Endometrial cancer, which is also referred to as womb or uterine cancer, is the most common gynaecological cancer in high-income countries. Endometrial cancer arises from the lining of the womb (endometrium). Over $90 \%$ of people with endometrial cancer are diagnosed following investigations for postmenopausal bleeding or heavy or irregular vaginal bleeding (Clarke 2018). It is estimated that $9 \%$ of those presenting with postmenopausal bleeding have an underlying endometrial cancer (Clarke 2018).

In the USA, more than 19 in every 100,000 women will be diagnosed with endometrial cancer each year. In comparison, only one to four per 100,000 women each year are diagnosed with this cancer in countries in Sub-Saharan Africa, the Middle East and South-Central Asia (Lortet-Tieulent 2018). Despite this variation, endometrial cancer is becoming more common throughout the world and has nearly overtaken cervical cancer to become the most common gynaecological cancer in middle-income countries (Bray 2018). This is thought to be due to the association of this cancer with excess body weight, later childbearing and smaller families. A small number of endometrial cancers (less than $5 \%$ ) are caused by an inheritable genetic condition, known as Lynch syndrome. People with Lynch syndrome have gene alterations that increase their risk of bowel, ovarian and endometrial cancer. People with this gene change will have a $30 \%$ to $60 \%$ lifetime risk of endometrial cancer.

The outlook for most people diagnosed with endometrial cancer is good. The majority (70\%) will present when the cancer is still confined to the womb (i.e. early-stage disease) and will be completely cured of their disease. Even when all stages of endometrial cancer are considered, nearly $80 \%$ of people affected can expect to survive for at least five years after their diagnosis, if they live in a high-income countries (ONS 2016).

Initial treatment for endometrial cancer usually involves surgery to remove the womb and other potentially affected organs. After surgery, these organs are examined by a pathologist to gain information about how far the cancer has spread (i.e. stage) and its type. This provides prognostic information, allowing an estimation to be made about how likely the cancer is to recur. Many of those affected will be cured completely by surgery alone and never require further treatment. However, approximately $10 \%$ to $15 \%$ of people with endometrial cancer will develop a recurrence (Bendifallah 2017; Salani 2017). The prognosis for those that develop recurrence is poor and most of these people will die from their disease (Bendifallah 2017). Being able to predict who these people might be offers the opportunity to prevent recurrence and potentially improve the rate of cure in this group. It also might allow us to identify those who are very unlikely to have recurrence and may be able to avoid additional treatment and its associated risks.

Additional treatment in the form of chemotherapy or radiotherapy (or both), sometimes referred to as adjuvant therapy, is offered to those deemed to be at higher risk of recurrence. This may include localised internal radiation applied to the top of the vagina (i.e. vaginal brachytherapy), radiation delivered from outside the body (i.e. external beam radiotherapy), drugs given to kill cancer cells (i.e. chemotherapy), or combinations of these. The most effective way of delivering this additional therapy, and the sequence in which it should be delivered, remains unclear (de Boer 2018; Galaal 2014; Matei 2019) All treatments are associated with potential side-effects, which may limit the tolerability of the treatment and cause long-term treatment-related side effects such as bowel and bladder problems, loss of sensation and vaginal discomfort (Ao 2020; de Boer 2016). Determining the optimal selection strategy will hopefully reduce under- and over-treatment in those diagnosed with endometrial cancer.

Additionally, being able to predict the risk of recurrence in people treated for endometrial cancer may allow for the tailoring of follow-up. In the UK, the British Gynaecological Cancer Society recommends that people who have completely recovered physically after their treatments and who are deemed at the lowest risk of recurrence (less than $10 \%$ risk of recurrence) can be empowered to contact their cancer care provider if they notice vaginal bleeding, new lumps or bumps or swelling of their abdomen, rather than attending routine follow-up appointments. This is known as patient-initiated follow-up (Newton 2020). This frees up time for healthcare providers to focus on providing individualised and holistic care for those who continue to have physical or psychological problems after treatment. Those at higher risk of recurrence can be offered either face-to-face or telephone follow-up before moving to patient-initiated follow-up two or more years post-treatment, as after this time the risk of recurrence declines (Newton 2020).

\section{Description of the prognostic models}

Rather than being a single disease, endometrial cancer should be thought of as a group of cancers that share a common origin, i.e. the womb lining. The different types of endometrial cancer are thought to develop through slightly different biological mechanisms (Hecht 2006; Piulats 2017). This may explain the differences seen in the disease courses and ultimately, why some people are more likely to develop a recurrence or die from their disease.

There are various models that try to group features of tumours and the people affected by them by their risk of developing a recurrence or dying from the disease. The most predictive individual feature is the extent of spread of the disease at the time of diagnosis (i.e. the stage). The International Federation of Gynecology and Obstetrics (FIGO) staging system is the most commonly used system to describe the extent of spread in endometrial cancer. It provides a common framework to describe spread of disease and is regularly updated to reflect the evolving understanding of the relative importance of different findings (Creasman 2009; Pecorelli 2009). The extent of spread (stage) is classified from 1 to 4 , with 1 being the earliest stage and having the lowest likelihood of recurrence and death; and 4 being the most advanced stage, with the highest likelihood of recurrence and death. In the last update in 2009 , some of the substages in early-stage endometrial cancer were removed, as no significant difference was seen in survival of people in these groups. Additionally, spread of the cancer to the glandular tissue of the cervix was downgraded to Stage 1 and the presence of free-floating cancer cells in the fluid surrounding the abdominal organs was no longer considered to provide additional information about the risk of recurrence.

Other recognised risk factors for recurrence include age, tumourrelated features, such as grade (i.e. how changed the cells appear), depth of invasion of the cancer into the muscle wall of the womb, lymphovascular space invasion (i.e. whether cancer cells 
are seen within the blood and lymph vessels in the womb), site of the tumour within the womb, tumour subtype and tumour size (Singh 2019). These factors have been combined into a number of different risk classification systems to help determine who might benefit from adjuvant therapy (Table 1). Although there is significant overlap between those classified at highest risk within all of the classifications systems, the incorporation of different risk factors into each model can lead to different conclusions in some cases. Additionally, because the classification and grading of womb cancer are very subjective, this can leads to disagreement in approximately one-third of endometrial cancers designated as high risk (Gilks 2013; Guan 2011; Han 2013 Hoang 2013). The poor performance of prognostic models based predominantly on features noted at pathological examination may, therefore, result in over- or under-treatment in a significant proportion of people with endometrial cancer.

Data from the Cancer Genome Atlas project demonstrated that endometrial cancer could be sub-categorised into four groups based upon distinct gene-level (i.e. molecular) alterations (Levine 2013). The presence of these features allows pathologists to objectively identify people with tumours that are likely to recur and then to die of their disease. This has driven the development of molecular classification systems in endometrial cancer (Talhouk $2015)$ that have been shown to be reproducible in various settings (Bosse 2018; Britton 2019; Kommoss 2018; Talhouk 2017; Travaglino 2020).

This review aims to evaluate the comparative performance of all current prognostic models to predict recurrence and survival in those receiving curative treatment for endometrial cancer. It is hoped that the results of this review will be used to continue refining tools to guide whether people with this cancer are offered adjuvant therapy in addition to surgery, and influence decisions regarding follow-up following initial treatment.

\section{Health outcomes}

- Recurrence within five years of diagnosis

- Recurrence at any time after diagnosis

- Death due to endometrial cancer

- Death from any cause after endometrial cancer

\section{Why it is important to do this review?}

With increasing numbers of people affected by endometrial cancer, and efforts to diagnose it at earlier stages, it will be increasingly important to provide accurate estimates of prognosis to guide treatment and follow-up decisions. The James Lind Alliance Womb Cancer Alliance project in 2016 identified that research to evaluate predictors of recurrence was amongst the top 30 research priorities for healthcare professionals and people affected by endometrial cancer (Wan 2016). It is important to evaluate whether the predictive performance of current risk models is adequate because these are widely used to guide treatment, and more recently to streamline follow-up. This topic is also particularly timely given the move towards clinical trials offering specific treatments to people with tumours that express particular molecular markers.

\section{O B J E C T I VES}

To review all prognostic models that combine two or more clinical, histological or molecular variables, or a combination of these variables, to provide an individualised assessment of risk of recurrence or death from disease and evaluate their performance to predict these outcomes in people undergoing curative treatment for endometrial cancer.

\section{METHODS}

\section{Criteria for considering studies for this review}

Please refer to Table 2 for the type of patients, interventions, comparators, outcomes, timings and settings (PICO) considered relevant to this review.

\section{Types of studies}

We will include all retrospective and prospective studies that evaluate the performance of prognostic models to predict recurrence or survival in people who have undergone curative treatment for endometrial cancer. We will only include studies where both surgical and histopathological features are used to describe the extent of spread of the disease (i.e. those published after the 1988 FIGO classification was introduced) to allow comparisons of this to other staging systems found within the searches. We will consider studies describing the development of a model with and without external validation. These studies will only be included if the model was applied to data that would be available following surgical staging but prior to adjuvant therapy and where there is follow-up of at least five years. Where external validation using an independent data set has not been applied, we will only include studies where there is evidence of appropriate adjustments made for over-optimism.

\section{Targeted population}

We will include studies in adults (18 years and over) with primary endometrial carcinomas of any histotype who have undergone surgery with curative intent. We will exclude studies or data pertaining to people with non-endometrial cancers that have metastasised to the body of the uterus, people with advanced endometrial cancers in whom surgical staging was not performed, people treated with chemotherapy, radiotherapy or hormonal therapy alone and people considered ineligible for surgery or who declined surgery.

\section{Types of prognostic predictive model}

We will assess all prognostic models that combine two or more clinical, histological or molecular variables, or a combination of these variables, and use a mathematical function to predict recurrence or survival in people with endometrial cancer. In this context, we define molecular variables as any recognised marker of gene function. These may include, but are not limited to: somatic or germline DNA variation, DNA methylation status, and protein expression.

\section{Types of outcomes to be predicted}

\section{Primary outcomes}

- Recurrence within five years of diagnosis: this outcome was chosen as the primary outcome as many of these models are used to determine the need for additional adjuvant therapy. In clinical practice, five years of follow-up is the current standard. Whilst survival may not be extended by adjuvant treatment (Johnson 2011; Kong 2012), the time in which the patient is free of cancer-related symptoms or requiring treatment (or both) is 
important in facilitating shared decision making, determining the efficacy of new treatment strategies, and planning provision of healthcare services for those treated for endometrial cancer.

- Recurrence at any time following initial treatment: we acknowledge that although $80 \%$ of recurrences occur within the first three years after treatment, a small subgroup of people with endometrial cancer will develop later recurrences. These people may have disease characteristics that put them at higher risk of late recurrences. Predicting recurrence at any time point is therefore also important, but will be considered separately.

- All-cause mortality: we will consider all-cause mortality as this has implications on shared decision-making and planning of broader healthcare services.

- Cancer-specific mortality: we will consider cancer-specific mortality separately as this has implications for assessing the efficacy of cancer treatments and for planning cancer services.

\section{Search methods for identification of studies}

\section{Electronic searches}

We will perform electronic searches of the following databases and sources.

\section{- Ovid MEDLINE \\ - Ovid Embase \\ - Web of Science}

As no specific search filters exist to identify prognostic model studies, we will combine and modify search filters suggested by Geersing 2012 and Pace 2014 to formulate a search strategy to identify models that seek to predict recurrence or survival (or both) in people with endometrial cancer. We will ensure that this is returning appropriate results by screening the MEDLINE searches and adapting this as appropriate, before translating these searches for use in the other sources (see Appendix 1).

In 1988, FIGO staging switched from a clinical staging system to a system that incorporated both surgical and histopathological findings; therefore, we will only include studies published after 1988 and up to the present day to allow comparisons of this to other staging systems found within the searches. We will include studies published in all languages and where these are published in languages other than English, we will have these translated as appropriate to reduce language bias. For all included studies, we will check for retractions and corrections. If there are more than twelve months between our original search and publication of the review, we will perform a further search to check for new studies prior to submission.

\section{Searching other resources}

In addition to the electronic searches outlined above, we will cross-check the reference lists of the retrieved articles, relevant reviews and guidelines pertaining to the management of endometrial cancer published by national and international specialist societies such as, but not limited to, the Society of Gynecologic Oncology, the European Society of Gynaecological Oncology, the European Society of Medical Oncology. We will search grey literature including Open Grey, ProQuest Dissertations \& Theses Global, Databases of ongoing trials that involve the metaRegister of Controlled Trials (mRCT) (www.isrctn.com/page/ $\mathrm{mrct}$ ) and ClinicalTrials.gov. We intend to contact authors of relevant studies, experts, and investigators who are known to be active in the field for further information on ongoing studies.

\section{Data collection}

\section{Selection of studies}

The titles and abstracts of all potentially eligible studies identified through the searches will be screened in duplicate by teams of two independent review authors (AM, EB, BR, IES, YLW, TDJW). We will retrieve the full-text study reports of all records considered potentially eligible by any review author. The full reports of those studies identified at the initial screening will be independently reviewed by two review authors (YLW, IES). These review authors will make a recommendation regarding which of these studies should be included and record the reason for exclusion for any ineligible studies. A third review author (EC) will resolve any disagreements through discussion amongst all three review authors. If no agreement can be reached, then the wider review author group will be consulted. The decisions made regarding the studies for inclusion will be collated using the author-support tool, Covidence (Covidence 2017).

We will remove duplicates and collate multiple reports from the same study into a single record. We will document the total number of retrieved records and included and excluded studies using a flow diagram, as recommended by the Preferred Reporting Items for Systematic Reviews and Meta-Analyses (PRISMA) statement (Page 2021). We will also complete 'Characteristics of excluded studies' tables.

\section{Data extraction and management}

Data extraction will be based on the 'CHecklist for critical Appraisal and data extraction for systematic Reviews of prediction Modelling Studies' (CHARMS) checklist (Moons 2014). We will use a standardised data collection form to record the data extracted agreed upon by the study team; we will pilot this form on three included studies (see Appendix 2).

\section{Assessment of risk of bias in included studies}

We intend to classify each study into one of the three categories (model development with or without external validation in the same publication and external validation study of a previously developed model only); we will assess risk of bias for each developed or validated model and for each outcome predicted by the model. We will adapt the 'Prediction model Risk Of Bias ASsessment Tool' (PROBAST) to assess risk of bias for each developed or validated model, for each outcome (Wolff 2019). PROBAST is structured around the assessment of four key domains, as follows.

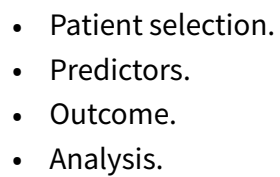

Each domain is judged for risk of bias (low, high or unclear); signalling questions for each domain facilitate this (see Appendix 3). Signalling questions are rated as yes $(\mathrm{Y})$, probably yes (PY), probably no $(\mathrm{PN})$, no $(\mathrm{N})$ or no information (NI). All signalling questions are phrased so that "yes" indicates the absence of bias. Any signalling question rated as "no" or "probably no" indicates the 
potential for bias. Two review authors (IES, EB) will independently make a judgement to determine whether each domain should be rated as "high", "low" or "unclear" risk of bias.

Prognostic model evaluation is judged to be at low risk of bias if all domains were rated as being at low risk of bias or if the prediction model was based on a very large data set and included some form of internal validation. However, if a prognostic model was developed without any external validation, and it was rated as being at low risk of bias for all domains, we will consider categorising it as high risk of bias. If at least one domain is judged to be at high or unclear risk of bias, then prognostic model evaluation is judged to be at high or unclear risk of bias.

The first three domains are also rated for concerns regarding the applicability of the model to the research question (low/ high/unclear). If there are low concerns about applicability for all domains, the prediction model evaluation is judged to have low concerns otherwise; if at least one domain is judged to have high or unclear concerns, then prediction model evaluation is judged to have high or unclear concerns about applicability, respectively. We intend to resolve any discrepancy by discussion and involvement of a third independent review author to solve any disagreement. Should there be insufficient information to make a judgement, we intend to contact the corresponding authors via email to request additional information to be able to make sound judgements on the 'Risk of bias' assessment.

\section{Measures of association or predictive performance measures to be extracted}

We will follow the guidance for meta-analysing the predictive performance of prognostic models and will extract the performance measures with associated precision measures (standard error or confidence interval) for each studied model from the development and their corresponding validation studies (Debray 2017). Two main statistical measures will be extracted: discrimination and calibration. Discrimination refers to model's ability to distinguish between individuals who develop and do not develop the outcome. The concordance (C) statistic is the most commonly used discrimination measure. It ranges from 0.5 (no discriminative ability) to 1 (perfect discriminative ability) (Snell 2018). For survival models that assess time to recurrence or death, the ' $D$ ' statistic will be extracted. The $\mathrm{D}$ statistic is a discrimination measure that gives the log hazard ratio of a model's predicted risks, dichotomised at the median value. Calibration refers to a model's accuracy of predicted risk probabilities and indicates the extent to which expected outcomes (predicted from the model) and observed outcomes agree. We will extract the following calibration performance measures: calibration plots (expected probabilities plotted against observed outcome frequencies), calibration slopes and observed-to-expected ratios (O:E ratios, ratios between observed and expected numbers of events or outcome frequencies) (Moons 2015).

\section{Unit of analysis issues}

We will compare the institutions and year of inclusion of all validation cohorts belonging to a model to determine whether there is a potential overlap in participants. When the same cohort was used to validate two or more different prognostic models, we will consider them as separate studies.

\section{Dealing with missing data}

We intend to contact the corresponding authors of primary studies to provide information on missing data for our analyses. In case of any non-response, we can estimate performance measures and standard errors such as the O:E ratio and C statistic, using formulas described by Debray and colleagues (Debray 2017; Debray 2018). If data are still insufficient, we will conduct a sensitivity analysis to explore the impact of the missing data.

\section{Assessment of heterogeneity}

We will investigate potential sources of clinical and statistical heterogeneity based on extracted items mentioned in the section "Data extraction and data management", according to the CHARM checklist. We plan to inspect forest plots to assess between-study heterogeneity and calculate tau 2,12 and prediction intervals which provide a range for the potential performance in a new validation study (Riley 2011), although this may be very wide if less than 10 studies are included. We will also conduct subgroup analyses to explore potential sources of heterogeneity. Specific attention will be paid to the year of diagnosis, as FIGO staging for this condition changed in 2009 as discussed in the Background. Heterogeneity is expected to arise due to differences in treatment regimens undergone by the participants in the different studies.

\section{Assessment of reporting deficiencies}

The majority of studies reporting on prognostic models are not prospectively registered and do not have published protocols (Peat 2014); subsequently, assessment of potential publication bias is difficult. If a study protocol is available, we will check articles for protocol violations.

Calibration measures, especially calibration slope and plot, are also not commonly reported (Bouwmeester 2012) despite the existence of methodological and reporting recommendations, i.e. the TRIPOD statement (Transparent Reporting of a multivariable prediction model for Individual Prognosis or Diagnosis). This may limit the reliability and applicability of many studies (Bouwmeester 2012). Where available, we will report the calibration measures used, and will make it clear in the review when these were not available.

\section{Data synthesis}

\section{Data synthesis and meta-analysis approaches}

We will consider conducting a meta-analysis of a model's discrimination and calibration performance if it has been developed or validated at least three times for the outcome in question in independent data sets. We will pool only validation studies of the outcome that a model was developed for. We will perform Logit transformation for the $C$ statistic and the log transformation for O:E ratio to improve normal between-study distribution (Snell 2018). If meta-analysis is performed, a randomeffects approach is essential to allow for unexplained heterogeneity across studies. We will perform meta-analyses through methods proposed by Debray 2017, using the meta-analysis packages in the $\mathrm{R}$ statistics language, which include metafor and metamisc (metamisc 2020).

Where the $\mathrm{C}$ statistic is not reported, we will not estimate it. Should the $C$ statistic be provided without measures of uncertainties, we will calculate the standard error and confidence intervals based 
on the $P$ value or the combination of sample size and the number of events, if available, according to the recommendations of Newcombe and colleagues (Debray 2017; Debray 2018; Newcombe 2006).

\section{Subgroup analysis and investigation of heterogeneity}

A subgroup analysis will be performed to investigate whether the change in FIGO staging over time has affected the performance of the models. We will conduct meta-regression, if needed, which extends a standard random-effects meta-analysis model by including study-level covariates. The dependent variable is the (transformed) estimate of the model performance measure (C statistic or O:E ratio) (Deeks 2021). Study-level or summarised patient-level data then will be used as explanatory or independent variables. Meta-regression explores the cause and extent of the between-study heterogeneity (Riley 2011). However, if the number of studies less than 10 , the power to detect a genuine association with meta-regression will usually be low.

\section{Sensitivity analysis}

We will perform sensitivity analyses by excluding studies with high risk of bias for at least one domain, studies in people receiving adjuvant therapy, and studies with missing data.

\section{Conclusions and summary of findings}

We will use the definitions laid out by the GRADE Working Group and the GRADE checklist to evaluate levels of certainty (Meader
2014). This will be presented in the form of a tabulated summary of our findings, generated in GRADEpro GDT (GRADEpro GDT; Meader 2014; Mustafa 2013); see Appendix 4. Currently there is no GRADE assessment specifically for prognostic model studies. However, if GRADE guidelines for prognostic model studies are published before we start analysing our results, we will use that guidance instead.

\section{ACKNOWLEDGEMENTS}

We would like to thank Jo Morrison for clinical and editorial advice; Jo Platt for designing the search strategy; Gail Quinn, Clare Jess and Tracey Harrison for their contribution to the editorial process; and the Cochrane Prognosis Methods Group for their assistance and support in preparing this review.

This project was supported by the National Institute for Health Research (NIHR), via Cochrane Infrastructure, Cochrane Programme Grant or Cochrane Incentive funding to the Cochrane Gynaecological, Neuro-oncology and Orphan Cancer Group. The views and opinions expressed therein are those of the authors and do not necessarily reflect those of the Systematic Reviews Programme, NIHR, NHS or the Department of Health.

The authors and Cochrane Gynaecological, Neuro-oncology and Orphan Cancers Team are very grateful to the following peer reviewers for their time and comments: Katharine Edey, Chumnan Kietpeerakool, Jayne Lawson and Hans Nagar. 


\section{RE F E R E N C E S}

\section{Additional references}

\section{Ao 2020}

Ao M, Ding T, Tang D, Xi M. Efficacy and toxicity of adjuvant therapies for high-risk endometrial cancer in stage I-III: a systematic review and network meta-analysis. Medical Science Monitor 2020;26:e925595-1-15.

\section{Bendifallah 2014}

Bendifallah S, Canlorbe G, Raimond E, Hudry D, Coutant C, Graesslin O, et al. A clue towards improving the European Society of Medical Oncology risk group classification in apparent early stage endometrial cancer? Impact of lymphovascular space invasion. British Journal of Cancer 2014;110(11):2640-6.

\section{Bendifallah 2017}

Bendifallah S, Ouldamer L, Lavoue V, Canlorbe G, Raimond E, Coutant $\mathrm{C}$, et al. Patterns of recurrence and outcomes in surgically treated women with endometrial cancer according to ESMO-ESGO-ESTRO Consensus Conference risk groups: Results from the FRANCOGYN study Group. Gynecologic Oncology 2017;144(1):107-112.

\section{Bosse 2018}

Bosse T, Nout RA, McAlpine JN, McConechy MK, Britton H, Hussein YR, et al. Molecular classification of grade 3 endometrioid endometrial cancers identifies distinct prognostic subgroups. American Journal of Surgical Pathology 2018;42(5):561-8.

\section{Bouwmeester 2012}

Bouwmeester W, Zuithoff NP, Mallett S, Geerlings MI, Vergouwe Y, Steyerberg E, et al. Reporting and methods in clinical prediction research: a systematic review. PLoS Medicine 2012;9(5):1-12.

\section{Bray 2018}

Bray F, Ferlay J, Soerjomataram I, Siegel RL, Torre LA, Jemal A. Global cancer statistics 2018: GLOBOCAN estimates of incidence and mortality worldwide for 36 cancers in 185 countries. CA: A Cancer Journal for Clinicians 2018;68(6):394-424.

\section{Britton 2019}

Britton H, Huang L, Lum A, Leung S, Shum K, Kale M, et al. Molecular classification defines outcomes and opportunities in young women with endometrial carcinoma. Gynecologic Oncology 2019;153(3):487-95.

\section{Clarke 2018}

Clarke MA, Long Beverly J, Del Mar MA, Arbyn M, BakkumGamez JN, Wentzensen N. Association of endometrial cancer risk with postmenopausal bleeding in women: a systematic review and meta-analysis. JAMA Internal Medicine 2018;178(9):1210-22.

\section{Colombo 2013}

Colombo N, Preti E, Landoni F, Carinelli S, Colombo A, Marini C, et al. Endometrial cancer: ESMO Clinical Practice Guidelines for diagnosis, treatment and follow-up. Annals of Oncology 2013;24:vi33-8.

\section{Covidence 2017 [Computer program]}

Veritas Health Innovation Covidence. Melbourne, Australia: Veritas Health Innovation, accessed prior to 14 January 2021.

\section{Creasman 2009}

Creasman W. Revised FIGO staging for carcinoma of the endometrium. International Journal of Gynaecology and Obstetrics 2009;105(2):109.

\section{Creutzberg 2000}

Creutzberg CL, van Putten WL, Koper PC, Lybeert ML, Jobsen JJ, Wárlám-Rodenhuis CC, et al. Surgery and postoperative radiotherapy versus surgery alone for patients with stage-1 endometrial carcinoma: multicentre randomised trial. PORTEC Study Group. Post Operative Radiation Therapy in Endometrial Carcinoma. Lancet 2000;355(9213):1404-11.

\section{de Boer 2016}

de Boer S M, Powell M E, Mileshkin L, Katsaros D, Bessette P, Haie-Meder C, et al. Toxicity and quality of life after adjuvant chemoradiotherapy versus radiotherapy alone for women with high-risk endometrial cancer (PORTEC-3): an openlabel, multicentre, randomised, phase 3 trial. Lancet Oncology 2016;17(8):1114-26.

\section{de Boer 2018}

de Boer S M, Powell M E, Mileshkin L, Katsaros D, Bessette P, Haie-Meder $C$, et al. Adjuvant chemoradiotherapy versus radiotherapy alone for women with high-risk endometrial cancer (PORTEC-3): final results of an international, openlabel, multicentre, randomised, phase 3 trial. Lancet Oncology 2018;19(3):295-309.

\section{Debray 2017}

Debray TPA, Damen JAAG, Snell KIE, Ensor J, Hooft L, Reitsma JB, et al. A guide to systematic review and metaanalysis of prediction model performance. The BMJ 2017;356:i6460.

\section{Debray 2018}

Debray TPA, Damen JAAG, Riley RD, Snell K, Reitsma JB, Hooft L, et al. A framework for meta-analysis of prediction model studies with binary and time-to-event outcomes. Statistical Methods in Medical Research 2018;28(9):2768-86.

\section{Deeks 2021}

Deeks JJ, Higgins JPT, Altman DG (editors). Chapter 10: Analysing data and undertaking meta-analyses. In: Higgins JPT, Thomas J, Chandler J, Cumpston M, Li T, Page MJ, Welch VA (editors). Cochrane Handbook for Systematic Reviews of Interventions version 6.2 (updated February 2021).

\section{Galaal 2014}

Galaal K, Al Moundhri M, Bryant A, Lopes AD, Lawrie TA. Adjuvant chemotherapy for advanced endometrial cancer. 
Cochrane Database of Systematic Reviews 2014, Issue 5. Art. No: CD010681. [DOI: 10.1002/14651858.CD010681.pub2]

\section{Geersing 2012}

Geersing GJ, Bouwmeester W, Zuithoff P, Spijker R, Leeflang M, Moons K G, et al. Search filters for finding prognostic and diagnostic prediction studies in Medline to enhance systematic reviews. PloS One 2012;7(2):e32844.

\section{Gilks 2013}

Gilks CB, Oliva E, Soslow RA. Poor interobserver reproducibility in the diagnosis of high-grade endometrial carcinoma. American Journal of Surgical Pathology 2013;37(6):874-81.

\section{GRADEpro GDT [Computer program]}

(developed by Evidence Prime, Inc.) GRADEpro Guideline Development Tool. McMaster University: (developed by Evidence Prime, Inc.), 2020. Available from gradepro.org.

\section{Guan 2011}

Guan H, Semaan A, Bandyopadhyay S, Arabi H, Feng J, Fathallah L, et al. Prognosis and reproducibility of new and existing binary grading systems for endometrial carcinoma compared to FIGO grading in hysterectomy specimens. International Journal of Gynecological Cancer 2011;21(4):654-60.

\section{Han 2013}

Han G, Sidhu D, Duggan MA, Arseneau J, Cesari M, Clement PB, et al. Reproducibility of histological cell type in high-grade endometrial carcinoma. Modern Pathology 2013;26(12):1594-604.

\section{Hecht 2006}

Hecht JL, Mutter GL. Molecular and pathologic aspects of endometrial carcinogenesis. Journal of Clinical Oncology 2006;24(29):4783-91.

\section{Hoang 2013}

Hoang LN, McConechy MK, Köbel M, Han G, Rouzbahman M, Davidson B, et al. Histotype-genotype correlation in 36 highgrade endometrial carcinomas. American Journal of Surgical Pathology 2013;37(9):1421-32.

\section{Johnson 2011}

Johnson N, Bryant A, Miles T, Hogberg T, Cornes P. Adjuvant chemotherapy for endometrial cancer after hysterectomy. Cochrane Database of Systematic Reviews 2011, Issue 10. Art. No: CD003175. [DOI: 10.1002/14651858.CD003175.pub2]

\section{Keys 2004}

Keys HM, Roberts JA, Brunetto VL, Zaino RJ, Spirtos NM, Bloss JD, et al. A phase III trial of surgery with or without adjunctive external pelvic radiation therapy in intermediate risk endometrial adenocarcinoma: a Gynecologic Oncology Group study. Gynecologic Oncology 2004;92(3):744-51.

\section{Kommoss 2018}

Kommoss S, McConechy MK, Kommoss F, Leung S, Bunz A, Magrill J, et al. Final validation of the ProMisE molecular classifier for endometrial carcinoma in a large populationbased case series. Annals of Oncology 2018;29(5):1180-8.

\section{Kong 2012}

Kong A, Johnson N, Kitchener HC, Lawrie TA. Adjuvant radiotherapy for stage I endometrial cancer. Cochrane Database of Systematic Reviews 2012, Issue 4. Art. No: CD003916. [DOI: 10.1002/14651858.CD003916.pub4]

\section{Levine 2013}

Levine DA, Cancer Genome Atlas Research Network. Integrated genomic characterization of endometrial carcinoma. Nature 2013;497(7447):67-73

\section{Lortet-Tieulent 2018}

Lortet-Tieulent J, Ferlay J, Bray F, Jemal A. International patterns and trends in endometrial cancer incidence, 1978-2013. Journal of the National Cancer Institute 2018;110(4):354-61.

\section{Matei 2019}

Matei D, Filiaci V, Randall ME, Mutch D, Steinhoff MM, DiSilvestro PA, et al. Adjuvant chemotherapy plus radiation for locally advanced endometrial cancer. New England Journal of Medicine 2019;380(24):2317-26.

\section{Meader 2014}

Meader N, King K, Llewellyn A, Norman G, Brown J, Rodgers M, et al. A checklist designed to aid consistency and reproducibility of GRADE assessments: development and pilot validation. Systematic Reviews 2014;3:82.

\section{metamisc 2020 [Computer program]}

Available at cran.r-project.org/ metamisc: Meta-Analysis of Diagnosis and Prognosis Research Studies. Debray T, de Jong V, Version 0.2.3. Available at cran.r-project.org/, 2020.

\section{Moons 2014}

Moons KG, de Groot JA, Bouwmeester W, Vergouwe Y, Mallett S, Altman DG, et al. Critical appraisal and data extraction for systematic reviews of prediction modelling studies: the CHARMS checklist. PLoS Medicine 2014;11(10):e1001744.

\section{Moons 2015}

Moons KGM, Altman DG, Reitsma JB, loannidis JPA, Macaskill P, Steyerberg, EW, et al. Transparent Reporting of a multivariable prediction model for Individual Prognosis Or Diagnosis (TRIPOD): explanation and elaboration. Annals of Internal Medicine 2015;162(1):W1-73.

\section{Mustafa 2013}

Mustafa RA, Santesso N, Brozek J, Akl EA, Walter SD, Norman G et al. The GRADE approach is reproducible in assessing the quality of evidence of quantitative evidence syntheses. Journal of Clinical Epidemiology 2013;66(7):736-42.

\section{Newcombe 2006}

Newcombe RG. Confidence intervals for an effect size measure based on the Mann-Whitney statistic. Part2: asymptotic methods and evaluation. Statistics in Medicine 2006;25(4):559-73. 


\section{Newton 2020}

Newton C, Nordin A, Rolland P, Ind T, Larsen-Disney P, Martin-Hirsch P, et al. British Gynaecological Cancer Society recommendations and guidance on patient-initiated followup (PIFU). International Journal of Gynecologic Cancer 2020;30(5):695-700.

\section{ONS 2016}

Office for National Statistics (ONS). Cancer survival in England: 2010-2014 and followed up to 2015. www.ons.gov.uk/releases/ cancersurvivalforadultsinengland2015 2016.

\section{Pace 2014}

Pace NL, Carlisle J, Eberhart LHJ, Kranke P, Trivella M, Lee A, et al. Prediction models for the risk of postoperative nausea and vomiting. Cochrane Database of Systematic Reviews 2014, Issue 9. Art. No: CD011318. [DOI: 10.1002/14651858.CD011318]

\section{Page 2021}

Page MJ, McKenzie JE, Bossuyt PM, Boutron I, Hoffmann TC, Mulrow CD et al. The PRISMA 2020 statement: an updated guideline for reporting systematic reviews. BMJ 2021;372:n71.

\section{Peat 2014}

Peat G, Riley RD, Croft P, Morley KI, Kyzas PA, Moons KGM, et al. Improving the transparency of prognosis research: the role of reporting, data sharing, registration, and protocols. PLOS Medicine 2014;11(7):e1001671.

\section{Pecorelli 2009}

Pecorelli S. Revised FIGO staging for carcinoma of the vulva, cervix, and endometrium. International Journal of Gynaecology and Obstetrics 2009;105(2):103-4.

\section{Piulats 2017}

Piulats JM, Guerra E, Gil-Martín M, Roman-Canal B, Gatius S, Sanz-Pamplona R, et al. Molecular approaches for classifying endometrial carcinoma. Gynecologic Oncology 2017;145(1):200-7.

\section{Riley 2011}

Riley RD, Higgins JPT, Deeks, JJ. Interpretation of random effects meta-analyses. BMJ 2011;342:d549.

\section{Salani 2017}

Salani R, Khanna N, Frimer M, Bristow RE, Chen LM. An update on post-treatment surveillance and diagnosis of recurrence in women with gynecologic malignancies: Society of Gynecologic Oncology (SGO) recommendations. Gynecologic Oncology 2017;146(1):3-10.

\section{Singh 2019}

Singh N, Hirschowitz L, Zaino R, Alvarado-Cabrero I, Duggan MA, Ali-Fehmi R, et al. Pathologic prognostic factors in endometrial carcinoma (other than tumor type and grade). International Journal of Gynecological Pathology 2019;38 Suppl 1:S93-113.

\section{Snell 2018}

Snell KIE, Ensor J, Debray TPA, Moons KGM, Riley RD. Metaanalysis of prediction model performance across multiple studies: which scale helps ensure between-study normality for the C-statistic and calibration measures? Statistical Methods in Medical Research 2018;27(11):3505-22.

\section{Stelloo 2015}

Stelloo E, Bosse T, Nout RA, Mackay HJ, Church DN, Nijman HW, et al. Refining prognosis and identifying targetable pathways for high-risk endometrial cancer; a TransPORTEC initiative. Modern Pathology 2015;28(6):836-44.

\section{Talhouk 2015}

Talhouk A, McConechy MK, Leung S, Li-Chang HH, Kwon JS, Melnyk N, et al. A clinically applicable molecular-based classification for endometrial cancers. British Journal of Cancer 2015;113(2):299-310.

\section{Talhouk 2017}

Talhouk A, McConechy MK, Leung S, Yang W, Lum A, Senz J, et al. Confirmation of ProMisE: a simple, genomics-based clinical classifier for endometrial cancer. Cancer 2017;123(5):802-13.

\section{Todo 2010}

Todo Y, Kato H, Kaneuchi M, Watari H, Takeda M, Sakuragi N. Survival effect of para-aortic lymphadenectomy in endometrial cancer (SEPAL study): a retrospective cohort analysis. Lancet 2010;375(9721):1165-72.

\section{Travaglino 2020}

Travaglino A, Raffone A, Mascolo M, Guida M, Insabato L, Zannoni GF, et al. TCGA molecular subgroups in endometrial undifferentiated/dedifferentiated carcinoma. Pathology Oncology Research: POR 2020;26(3):1411-6.

\section{Wan 2016}

Wan YL, Beverley-Stevenson R, Carlisle D, Clarke S, Edmondson RJ, Glover S, et al. Working together to shape the endometrial cancer research agenda: the top ten unanswered research questions. Gynecologic Oncology 2016;143(2):287-93.

\section{Wolff 2019}

Wolff RF, Moons KGM, Riley RD, Whiting PF, Westwood M, Collins GS, et al. PROBAST: a tool to assess the risk of bias and applicability of prediction model studies. Annals of Internal Medicine 2019;170(1):51-8.

\section{ADDITIONAL TABLES}

Table 1. Commonly used models to classify risk of recurrence in endometrial cancer

\begin{tabular}{|c|c|c|c|}
\hline $\begin{array}{l}\text { Risk classification models } \\
\text { in endometrial cancer }\end{array}$ & Reference & Prognostic factors included & $\begin{array}{l}\text { Risk categories from lowest } \\
\text { risk to highest }\end{array}$ \\
\hline
\end{tabular}

Prognostic models for predicting recurrence and survival in women with endometrial cancer (Protocol) 
Table 1. Commonly used models to classify risk of recurrence in endometrial cancer (Continued)
The International Federa-
Creasman 2009
Extent of tumour spread, spread to regional
Stages 1-4 tion of Gynecology and Ob- lymph nodes, metastasis to distant sites
stetrics (FIGO) staging

Post Operative Radiation
Therapy in Endometrial Car-
cinoma (PORTEC)-1 classifi-
cation

Creutzberg 2000

FIGO stage, tumour grade, histological type, extent of myometrial invasion, age $>60$

Low

Intermediate

High-intermediate

High

Gynecologic Oncology $\quad$ Keys 2004
Group (GOG) 99 classifica-
tion

FIGO stage, tumour grade, histological type, extent of myometrial invasion, LVSI, stratified age category (i.e. $<50,50$ to $69,>70$ )

Low

Low-intermediate

High-intermediate

High

\begin{tabular}{ll}
\hline $\begin{array}{l}\text { Survival effect of para- } \\
\text { aortic lymphadenectomy } \\
\text { (SEPAL) in Endometrial can- }\end{array}$ & Todo 2010 \\
cer classification & \\
\hline $\begin{array}{l}\text { European Society of Med- } \\
\text { ical Oncology (ESMO) classi- } \\
\text { fication }\end{array}$ & Colombo 2013 \\
& \\
\hline $\begin{array}{l}\text { ESMO-modified classifica- } \\
\text { tion }\end{array}$ & Bendifallah 2014 \\
& Bendifallah 2014 \\
& Bendifallah 2014 \\
& Bendifallah 2014 \\
& Bendifallah 2014 \\
& Bendifallah 2014
\end{tabular}
FIGO stage, tumour grade, histological type
(i.e. endometrioid or non-endometrioid), ex-
tent of myometrial invasion, LVSI
Low

Intermediate

High
FIGO stage, tumour grade, histological type (i.e. endometrioid or non-endometrioid), extent of myometrial invasion
Low

Intermediate

High
FIGO stage, tumour grade, histological type (i.e. endometrioid or non-endometrioid), extent of myometrial invasion, LVSI
ESMO low risk/LVSI absent

ESMO low risk/LVSI present

ESMO intermediate risk/LVSI absent

ESMO intermediate risk/LVSI present

ESMO high risk/LVSI absent

ESMO high risk/LVSI present

$\begin{array}{lll}\begin{array}{l}\text { The Cancer Genome Atlas } \\ \text { classification }\end{array} & \text { Levine } 2013 & \begin{array}{l}\text { Ultramutation of POLE proofreading gene, } \\ \text { microstatellite instability, DNA copy number }\end{array} \\ \text { Levine } 2013 & \end{array}$

Levine 2013

Levine 2013
POLE hypermutated

Microsatellite instability hypermutated

Copy number low

Copy number high

POLE proofreading-mutant tumours

Microsatellite instable tumours

No specific molecular profile (NSMP) tumours 
Table 1. Commonly used models to classify risk of recurrence in endometrial cancer (Continued)

p53-mutant tumours

\begin{tabular}{|c|c|c|c|}
\hline $\begin{array}{l}\text { Proactive Molecular Risk } \\
\text { Classifier for Endometrial } \\
\text { Cancer (ProMisE) classifica- } \\
\text { tion }\end{array}$ & $\begin{array}{l}\text { Talhouk } 2017 \\
\text { Talhouk } 2017\end{array}$ & $\begin{array}{l}\text { Ultramutation of POLE proofreading gene, } \\
\text { MMR IHC, p53 IHC }\end{array}$ & $\begin{array}{l}\text { POLE mutant } \\
\text { MMR IHC abnormal } \\
\text { p53 wild type } \\
\text { p53 abnormal }\end{array}$ \\
\hline
\end{tabular}

LVSI - lymphovascular space invasion, POLE - DNA polymerase epsilon, MMR IHC - mismatch repair immunohistochemistry p53 IHC - p53 immunohistochemistry

Table 2. PICO question to be considered

\begin{tabular}{ll}
\hline Characteristics & Details of what will be considered \\
\hline Population & Women with a new diagnosis of primary endometrial cancer \\
\hline Intervention & $\begin{array}{l}\text { All prognostic models that combine at least two clinical, histological and/or molecular variables to } \\
\text { predict recurrence or survival in the population }\end{array}$ \\
\hline Comparator & - \\
\hline Outcomes & Recurrence at any site primarily and secondarily, cancer-specific survival \\
\hline Timing & Baseline: moment when need for adjuvant therapy decided, i.e. directly following staging surgery \\
but prior to adjuvant therapy \\
Outcome: moment when recurrence diagnosed or death from endometrial cancer occurs
\end{tabular}

\section{AP PE N DICES}

\section{Appendix 1. MEDLINE Ovid search strategy}

1. exp Endometrial Neoplasms/

2. ((endomet ${ }^{\star}$ or uter $^{\star}$ or womb $\left.^{\star}\right)$ adj4 (cancer ${ }^{\star}$ or neoplas ${ }^{\star}$ or carcinom $^{\star}$ or adenocarcinom ${ }^{\star}$ or malignan ${ }^{\star}$ or tumor $^{\star}$ or tumour $\left.{ }^{\star}\right)$ ).ti,ab.

3. 1 or 2

4. validat\$.mp. or predict\$.ti. or rule\$.mp.

5. (predict\$ and (outcome\$ or risk\$ or models\$)).mp.

6. ((history or variable\$ or criteria or scor\$ or characteristic\$ or finding\$ or factor\$) and (predict\$ or model\$ or decision\$ or identif\$ or prognos\$)).mp.

7. decision\$.mp. and ((model\$ or clinical\$).mp. or LOGISTIC MODELS/)

8. (prognostic and (history or variable\$ or criteria $\$$ or scor $\$$ or characteristic $\$$ or finding $\$$ or factor $\$$ or model\$)).mp.

9. exp ROC Curve/

10. ("stratification" or "ROC Curve" or "discrimination" or "discriminate" or "c-statistic" or "c statistic" or "Area under the curve" or AUC or "calibration" or "indices" or "algorithm" or "multivariable").mp.

11. ((risk* or molecular or prognostic) adj3 (stratification or classification)).ti,ab.

12. ((clinical ${ }^{\star}$ or histopathological ${ }^{\star}$ or histological ${ }^{\star}$ or clinicopathological) adj5 (feature* or classification* or grading $^{\star}$ or model $\left.^{\star}\right)$ ).ti,ab.

13. (Post Operative Radiation Therapy in Endometrial Carcinoma or PORTEC or Gynecologic Oncology Group 99 classification or GOG 99 classification or "survival effect of para-aortic lymphadenectomy" or SEPAL or ESMO* or "ESMO-modified" or Cancer Genome Atlas Classification or TGCA* or TransPORTEC* or ProMisE*).mp.

14. 4 or 5 or 6 or 7 or 8 or 9 or 10 or 11 or 12 or 13

15. exp Recurrence/ 
16. exp Neoplasm Recurrence, Local/

17. (recurren* or progress ${ }^{\star}$ or relapse* or repeat $^{\star}$ or return*).ti,ab.

18. (prevent $^{\star}$ or limit ${ }^{\star}$ or control ${ }^{\star}$ ).ti,ab.

19. 17 and 18

20. surveillance.ti,ab.

21.15 or 16 or 19 or 20

22. 3 and 14 and 21

Appendix 2. CHARM data extraction checklist

\begin{tabular}{|c|c|}
\hline Domain & Key items \\
\hline Source of data & (e.g. cohort, case-control, randomised trial participants, or registry data) \\
\hline \multirow[t]{4}{*}{ Participants } & $\begin{array}{l}\text { Participant eligibility and recruitment method (e.g., consecutive participants, location, number of } \\
\text { centers, setting, inclusion and exclusion criteria) }\end{array}$ \\
\hline & Participant description \\
\hline & Details of treatments received, if relevant \\
\hline & Study dates (e.g. recruitment period, follow up period, length of follow-up) \\
\hline \multirow[t]{6}{*}{ Outcome(s) to be predicted } & Definition and method for measurement of outcome \\
\hline & Was the same outcome definition (and method for measurement) used in all patients? \\
\hline & Type of outcome (e.g. single or combined endpoints) \\
\hline & Was the outcome assessed without knowledge of the candidate predictors (i.e. blinded)? \\
\hline & Were candidate predictors part of the outcome (e.g. in panel or consensus diagnosis)? \\
\hline & Time of outcome occurrence or summary of duration of follow-up \\
\hline \multirow[t]{4}{*}{ Model Development } & Modelling method (e.g. logistic, survival, neural network, or machine learning techniques) \\
\hline & Modelling assumptions satisfied \\
\hline & $\begin{array}{l}\text { Method for selection of predictors for inclusion in multivariable modelling (e.g. all candidate pre- } \\
\text { dictors, pre-selection based on unadjusted association with the outcome) }\end{array}$ \\
\hline & $\begin{array}{l}\text { Method for selection of predictors during multivariable modelling (e.g. full model approach, back- } \\
\text { ward or forward selection) and criteria used (e.g. P value, Akaike Information Criterion) }\end{array}$ \\
\hline
\end{tabular}

Model Performance

Calibration (calibration plot, calibration slope, Hosmer-Lemeshow test) and Discrimination

(C-statistic, D-statistic, log-rank) measures with confidence intervals

Classification measures (e.g. sensitivity, specificity, predictive values, net reclassification improvement) and whether a-priori cut points were used

\section{Model Evaluation}

Method used for testing model performance: development dataset only (random split of data, resampling methods e.g. bootstrap or cross-validation, none) or separate external validation (e.g. temporal, geographical, different setting, different investigators)

In case of poor validation, whether model was adjusted or updated (e.g. intercept recalibrated, predictor effects adjusted, or new predictors added) 
Results Final and other multivariable models (e.g. basic, extended, simplified) presented, including predictor weights or regression coefficients, intercept, baseline survival, model performance measures (with standard errors or confidence intervals)

Any alternative presentation of the final prediction models, e.g. sum score, nomogram, score chart, predictions for specific risk subgroups with performance

Comparison of the distribution of predictors (including missing data) for development and validation datasets

\begin{tabular}{|c|c|}
\hline \multirow[t]{2}{*}{ Sample size } & Number of participants and number of outcomes/events \\
\hline & $\begin{array}{l}\text { Number of outcomes/events in relation to the number of candidate predictors (Events Per Vari- } \\
\text { able) }\end{array}$ \\
\hline \multirow[t]{3}{*}{ Missing data } & Number of participants with any missing value (include predictors and outcomes) \\
\hline & Number of participants with missing data for each model \\
\hline & Handling of missing data \\
\hline Interpretation and discussion & Interpretation of presented models \\
\hline
\end{tabular}

Comparison with other studies, discussion of generalisability, strengths and limitations.

\section{Appendix 3. 'Risk of bias' signaling questions for each domain}

- Patient selection

* What study design was used?

* Was the inclusion and exclusion of participants appropriate?

* Was participant selection similar to the development study?

- Predictors

* Were predictors defined and assessed in a similar way for all participants?

* Were predictor assessments made without knowledge of outcome data?

* Are all predictors available at the time the model is intended to be used?

- Outcome

* Was the outcome determined appropriately?

* Was a pre-specified or standard outcome definition used?

* Were predictors excluded from the outcome definition?

* Was the outcome defined and determined in a similar way for all participants?

* Was the outcome determined without knowledge of predictor information?

* Was the time interval between predictor assessment and outcome determination appropriate? 
- Analysis

* Were there a reasonable number of participants with the outcome?

* Were there a reasonable number of participants with the outcome?

* Were continuous and categorical predictors handled appropriately?

* Were all enrolled participants included in the analysis?

* Were participants with missing data handled appropriately?

* Was selection of predictors based on univariable analysis avoided?

* Were complexities in the data (e.g. censoring, competing risks, sampling of controls) accounted for appropriately?

* Were relevant model performance measures evaluated appropriately?

* Was model overfitting, underfitting and optimism in model performance accounted for?

* Do predictors and their assigned weights in the final model correspond to the results from multivariable analysis?

\section{Appendix 4. Draft 'Summary of findings' table}

\section{Prognostic models for predicting recurrence in women with endometrial cancer}

Population: people with a new diagnosis of primary endometrial cancer

Index model: all clinicopathological and molecular prognostic models used to predict recurrence and survival in the population

Comparator: all models will be compared to FIGO staging (2009)

Timing: recurrence within five years and at any time following diagnosis and death at any time following diagnosis

Setting: risk stratification for adjuvant therapy

\begin{tabular}{|c|c|c|c|c|c|}
\hline Outcomes & Measure & $\begin{array}{l}\text { No of partici- } \\
\text { pants } \\
\text { (studies) }\end{array}$ & $\begin{array}{l}\text { Summary } \\
\text { measure }\end{array}$ & $\begin{array}{l}\text { Pooled re- } \\
\text { sult( }(95 \% \mathrm{Cl})\end{array}$ & Comment \\
\hline
\end{tabular}

Recurrence within five years of diagnosis

Recurrence at any time following diagnosis

All-cause mortality

Cancer-specific mortality

GRADE Working Group grades of evidence

High quality: Further research is very unlikely to change our confidence in the estimate of effect.

Moderate quality: Further research is likely to have an important impact on our confidence in the estimate of effect and may change the estimate.

Low quality: Further research is very likely to have an important impact on our confidence in the estimate of effect and is likely to change the estimate.

Very low quality: We are very uncertain about the estimate.

\section{CONTRIBUTIONSOF AUTHORS}

YLW \& IES drafted the protocol, which was reviewed and agreed by all authors. The search strategy was developed by YLW, TDJW and AM with the assistance of Information Specialist, Jo Platt from the Cochrane Gynaecological, Neuro-oncology and Orphan Cancers group. All authors will contribute to the selection of the studies to include and TDJW, BR, IES, EB will extract data from studies and carry out the analysis. The analysis will be interpreted and the final review will be drafted by YLW, IES, RJE \& EJC. 


\section{DECLARATIONS OF INTEREST}

YLW: no conflicts of interests to declare IES: no conflicts of interests to declare TDJW: no conflicts of interests to declare BR: no conflicts of interests to declare EB: no conflicts of interests to declare RJE: no conflicts of interests to declare EJC: no conflicts of interests to declare

\section{SOURCES OF SUPPORT}

\section{Internal sources}

- None, Other

None

\section{External sources}

- Cancer Research UK, UK

AM is supported by Cancer Research UK (grant number C18281/A29019) 